\title{
PROPERTIES OF THE LIBBY-NOVICK BETA DISTRIBUTION WITH APPLICATION
}

\author{
ZAFAR IQBAL ${ }^{1}$, MUHAMMAD RASHAD ${ }^{2,3,}{ }^{*}$, MUHAMMAD HANIF $^{3}$ \\ ${ }^{1}$ Department of Statistics, Government College, Gujranwala, Pakistan \\ ${ }^{2}$ Superior University, Lahore, Pakistan \\ ${ }^{3}$ National College of Business Administration E Economics, Lahore, Pakistan
}

*Corresponding author: rashid.geostat@gmail.com

\begin{abstract}
The beta distribution is one of the most popular probability distributions with applications to real life data. In this paper, an extension of this distribution called the Libby-Novick Beta distribution (LNBD) which is believed to provide greater flexibility to model scenarios involving skew-normal data than original one. Analytical expressions for various mathematical properties along with characterization based on one truncated moment. The estimation of LNBD's parameters is undertaken using the method of maximum likelihood estimation. For illustration and performance evaluation of LNBD two real-life data sets adapted from earlier studies are used.
\end{abstract}

\section{INTRODUCTION}

In statistical distributions, a vast activity has been observed in generalizing classical distributions by adding more parameters to make them more flexible in analyzing empirical data. In the past few decades, a major research effort has been devoted to the study of skewsymmetric distributions. Such distributions have been constructed by adding a new parameter

Received May 22nd, 2020; accepted June 15th, 2020; published April 9th, 2021.

2010 Mathematics Subject Classification. 62E10.

Key words and phrases. Libby-Novick Beta distribution (LNBD); moment generating function; mean deviation; Bonferroni curve; Lorenze curve; Gini index truncated moment.

(C2021 Authors retain the copyrights of their papers, and all open access articles are distributed under the terms of the Creative Commons Attribution License. 
to probability density function (pdf) of the cumulative distribution function (cdf) of symmetric distribution, resulting in families of asymmetric more flexible distributions that are analytically more flexible.

A motivation for the [15] beta distribution is a three- parameter generalization of the usual two-parameter beta distribution. It is perhaps the simplest generalization of the twoparameter beta distribution that allows for significant additional flexibility.

\section{Mathematical Properties}

The $p d f$ of the Libby-Novick beta distribution (LNBD) is as under:

$$
f(x)=\frac{c^{a} x^{a-1}(1-x)^{b-1}}{\mathrm{~B}(a, b)[1-(1-c) x]^{a+b}} ; 0<x<1, a, b, c>0
$$

\subsection{Shape properties of the $p d f$}

The LNBD defined in (2.1) has real flexibility and it is shown through graphs w.r.t. some different combinations of values of the parameters.
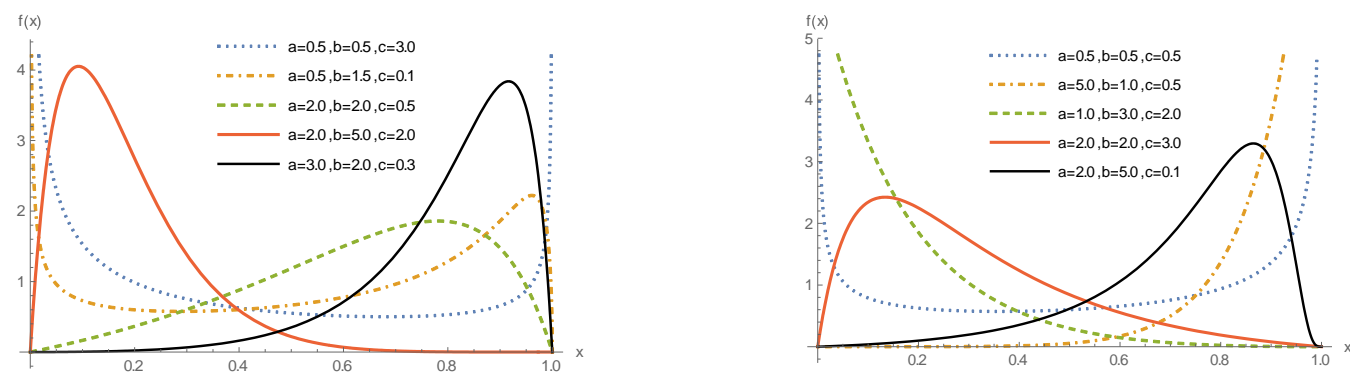

Figure 2.1

\section{Shapes of $p d f$}

(i) For $a>0, c>0$ and $0<b<1$ then LNBD is U-shaped.

(ii) For $0<a<1, b>1$ and $0<c<1$ then LNBD is S-shaped.

(iii) For $a=b=2, c \geq 1(c<1)$ and if $c \rightarrow \infty(c \rightarrow 0)$ then LNBD increases its positively skewed (negatively skewed) from the symmetric with decreasing mode (increasing mode).

(iv) For $a=b=c=1$, the LNBD is uniform distribution.

(v) For $a>2, b>2$ and $c \geq 1$ the LNBD is unimodel positively skewed with decreasing mode when $c \rightarrow \infty$.

(vi) For $a>2, b=1,0<c<1$, the form of LNBD is an increasing. 
(vii) For $a>2, b=1,1<c<3.5$, the LNBD increase but it increases slowly when $c$ increases in the interval and for $c \geq 3.5$ the LNBD again turns to unimodel.

(viii) For $b=c=1$, the LNBD is Power distribution.

(ix) For $a=c=1$, the LNBD is a special case of Kum-distribution or reflected exponentiated distribution.

2.2 Distribution function

The cumulative distribution function (CDF) of LNBD is

$$
\mathrm{F}(x)=\mathrm{I}_{c x /[1+(c-1) x]}(a, b)
$$

where $\mathrm{I}_{x}(a, b)=\frac{1}{\mathrm{~B}(a, b)} \int_{0}^{x} \mathrm{y}^{a-1}(1-\mathrm{y})^{b-1} \mathrm{dy}$
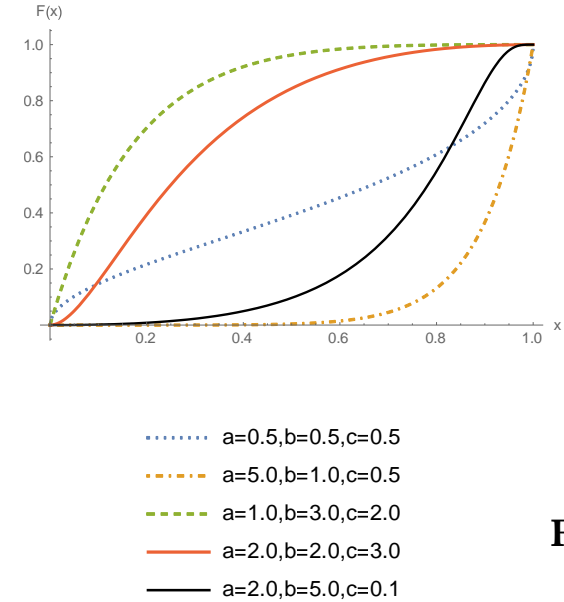

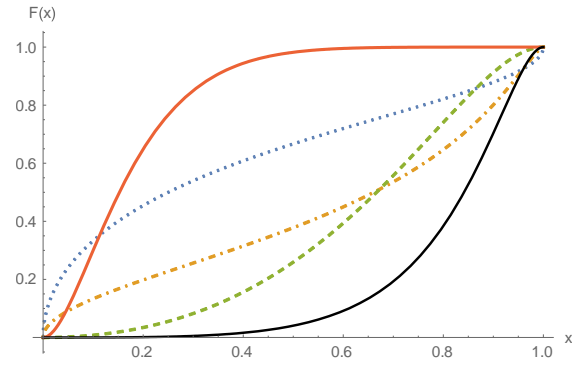

Figure 2.2

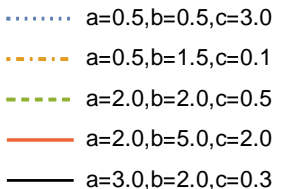

\subsection{Quantile function}

The Quantile function is given by:

$\mathrm{F}(x)=p \quad$ where $\quad 0<p<1$

$\mathrm{I}_{c x /[1+(c-1) x]}(a, b)=p$

and is describe as an inverse of $c d f$, as follows

$$
\begin{aligned}
x & =\frac{I^{-1}(p, a, b)}{c+(1-c) I^{-1}(p, a, b)} \\
& =\frac{Q(p ; a, b)}{c+(1-c) Q(p ; a, b)}
\end{aligned}
$$


where $Q(p ; a, b)$ is the inverse of beta function or quantile of beta function at $p$, and it can be numerically solved through software for different set of parameters' values. The graph can also be used to illustrate the behaviour of quantile function of LNBD.

i. For $a>1, b>1$ by increasing $c$, the quantile value of the LNBD decreases comparatively.

ii. For $a<1, c>1$, and for any value of $b$, the quantile value of the LNBD increases slowly for $p<0.5$ and for $p>0.5$, the quantile value sharply increases.

iii. For $c \rightarrow 0$, the quantile value of the LNBD increases sharply.

\subsection{Averages}

\subsubsection{Harmonic Mean}

The harmonic mean is of the $p d f(2.1)$ is

$$
H_{X}=\frac{a-1}{a+b c-1}
$$

The following are the limiting cases of $\mathrm{H}_{X}$ :

$\lim _{a \rightarrow 0} H_{X}$ is not defined.

$$
\begin{aligned}
& \lim _{a \rightarrow 1} H_{X}=\lim _{b \rightarrow \infty} H_{X}=0 \\
& \lim _{a \rightarrow \infty} H_{X}=\lim _{b \rightarrow 0} H_{X}=1
\end{aligned}
$$

\section{Corollary}

(i) If $c=1$ then $H_{X}=\frac{a-1}{a+b-1}$ is the harmonic mean of beta distribution.

(ii) If $a=b \& c=1$ then $\quad H_{X}=\frac{a-1}{2 a-1}$

and $\quad H_{1-X}=\frac{b-1}{c a+b-1}$

The following are the limiting cases of $H_{1-X}$ :

$\lim _{b \rightarrow 0} H_{1-X}$ is not defined. 


$$
\begin{aligned}
& \lim _{b \rightarrow 1} H_{1-X}=\lim _{a \rightarrow \infty} H_{1-X}=0 \\
& \lim _{a \rightarrow 0} H_{1-X}=\lim _{b \rightarrow \infty} H_{1-X}=1
\end{aligned}
$$

\section{Corollary}

(iii) If $c=1$ then $H_{1-X}=\frac{b-1}{a+b-1}$

(iv) If $a=b \& c=1$ then $\quad H_{1-X}=\frac{a-1}{2 a-1}$

A relation between $H_{X}$ and $H_{1-X}$ is as:

$$
H_{X}=H_{1-X}-\frac{c(a-b)(a+b-1)}{(a+b c-1)(a c+b-1)}
$$

when the $c=1$ then $H_{X}=H_{1-X}=\frac{a-b}{a+b c-1}$

\subsubsection{Geometric Mean}

The geometric mean $G_{X}$ of the $p d f(2.1)$ is

$$
G_{X}=\exp \left[\psi(a)-\psi(a+b)-\ln c+\sum_{i=1}^{\infty} \frac{1}{i}\left(1-\frac{1}{c}\right)^{i} \frac{B(a+i, b)}{B(a, b)}\right]
$$

and

$$
G_{1-X}=\exp \left[\frac{1}{B(a, b)} \sum_{i=0}^{\infty} \frac{B(a+i, b)}{i}\left\{\left(1-\frac{1}{c}\right)^{i}-1\right\}\right]
$$

\subsubsection{Mode}

Mode is obtained by solving $\quad \frac{d}{d x} f(x)=0$

$f(x)=\frac{c^{a} x^{a-1}(1-x)^{b-1}}{B(a, b)[1-(1-c) x]^{a+b}}$

$\ln f(x)=\ln C+(a-1) \ln x+(b-1) \ln (1-x)-(a+b) \ln [1-(1-c) x] \quad$ where $C=\frac{c^{a}}{B(a, b)}$ 


$$
\begin{aligned}
& \frac{d}{d x} \ln f(x)=\frac{a-1}{x}-\frac{b-1}{1-x}+\frac{(a+b)(1-c)}{1-(1-c) x} \\
& \frac{d}{d x} \ln f(x)=0 \\
& \frac{(a-1)(1-x)[1-(1-c) x]-(b-1) x[1-(1-c) x]+(a+b)(1-c) x(1-x)}{x(1-x)[1-(1-c) x]}=0 \\
& 2(c-1) x^{2}+(3-a-c-b c) x+(a-1)=0 \\
& x=\frac{-B \pm \sqrt{B^{2}-4 A C}}{2 A}
\end{aligned}
$$

$x=d$ when the quadratic equation cuts to $\mathrm{X}-$ axis.

$B^{2}-4 A C \geq 0$

$(3-a-c-b c)^{2}-8(a-1)(c-1) \geq 0$

$a^{2}+(1+b)^{2} c^{2} \geq 6 c(a+b)+2 a(1+c)$

Table 2.1 The Mode for the LNBD (For different values)

\begin{tabular}{cccc}
\hline $\mathrm{a}$ & $\mathrm{b}$ & $\mathrm{c}$ & Mode \\
\hline 1 & 1.1 & 0.1 & 0.99444 \\
1 & 1.2 & 0.2 & 0.97500 \\
1 & 1.3 & 0.3 & 0.93557 \\
1 & 1.4 & 0.4 & 0.86667 \\
1 & 1.5 & 0.5 & 0.75000 \\
1 & 1.6 & 0.6 & 0.55000 \\
1 & 1.7 & 0.7 & 0.18333 \\
1 & 1.8 & 0.8 & 0
\end{tabular}

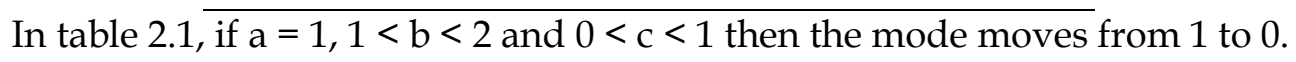


Table 2.2 The Mode for the LNBD (For different values)

\begin{tabular}{cccc}
\hline $\mathrm{a}$ & $\mathrm{b}$ & $\mathrm{c}$ & Mode \\
\hline 0.1 & 0.1 & 0.1 & 0.45779 \\
0.2 & 0.1 & 0.2 & 0.41890 \\
0.3 & 0.1 & 0.3 & 0.38120 \\
0.4 & 0.1 & 0.4 & 0.34322 \\
0.5 & 0.1 & 0.5 & 0.30371 \\
0.6 & 0.1 & 0.6 & 0.26127 \\
0.7 & 0.1 & 0.7 & 0.21405 \\
0.8 & 0.1 & 0.8 & 0.15920 \\
0.9 & 0.1 & 0.9 & 0.09160
\end{tabular}

In table 2.2, if $0<\mathrm{a}<1, \mathrm{~b}=0.1$ and $0<\mathrm{c}<1$ then the mode moves from 0.45 to 0.09 .

\section{$2.5 \quad$ Mean deviations}

The Mean Deviation of the LNBD from arithmetic mean and median are denoted by $\delta_{1}(Y)$ and $\delta_{2}(Y)$ respectively and are found from

\subsubsection{From Mean}

$$
\begin{aligned}
& \delta_{1}(Y)=\int_{0}^{1}|y-\mu| f(y) d y \\
& =2 \mu F(\mu)-2 J(\mu) \\
& =2 \mu I_{c \mu /[1+(c-1) \mu]}(a, b)-\frac{2}{c B(a, b)} \sum_{i=0}^{\infty} \frac{\Gamma(n+i)}{\Gamma(n)}\left(1-\frac{1}{c}\right)^{i} B_{c \mu /[1+(c-1) \mu]}(a+i+1, b)
\end{aligned}
$$

\subsubsection{From Median}

$$
\begin{aligned}
\delta_{2}(Y) & =\int_{0}^{1}|y-M| f(y) d y \\
& =\mu-2 J(M) \\
& =\mu-\frac{2}{c B(a, b)} \sum_{i=0}^{\infty} \frac{\Gamma(n+i)}{\Gamma(n)}\left(1-\frac{1}{c}\right)^{i} B_{c M /[1+(c-1) M]}(a+i+1, b)
\end{aligned}
$$




\section{$2.6 \quad r^{\text {th }}$ Moment}

The $r^{\text {th }}$ moments about origin of the $p d f(2.1)$ is

$$
E\left(X^{r}\right)=\frac{1}{c^{r} B(a, b)} \sum_{i=0}^{\infty} \frac{\Gamma(r+i)}{i ! \Gamma(r)}\left(1-\frac{1}{c}\right)^{i} B(a+r+i, b)
$$

\section{Corollary}

- If $c=1$, then $E(X)=\frac{a}{a+b}, E\left(X^{2}\right)=\frac{a(a+1)}{(a+b)(a+b+1)}, V(X)=\frac{a b}{(a+b)^{2}(a+b+1)}$

- If $a=b \& c=1$ then $E(X)=\frac{1}{2}$

\subsection{Moment generating function}

The moment generating function of the $p d f(2.1)$ about zero is

$$
M_{x}(t)=\frac{1}{B(a, b)} \sum_{r=0}^{\infty} \sum_{i=0}^{\infty} \frac{\Gamma(n+i)}{c^{r} \Gamma(n)}\left(1-\frac{1}{c}\right)^{i} B(a+r+i, b) \frac{\left(t^{r}\right)}{r !}
$$

\subsection{Factorial Moments}

\subsubsection{Decreasing Factorial Moments of LNBD}

The decreasing factorial moments of the LNBD is defined as:

$$
\begin{aligned}
E\left[(X)_{r}\right] & =E\left(\prod_{i=1}^{r} X-i+1\right) \\
& =\sum_{\mathrm{r}=0}^{n} S(n, r) E\left(X^{r}\right) \\
& =\frac{1}{B(a, b)} \sum_{\mathrm{r}=0}^{n} \sum_{i=0}^{\infty} \frac{S(n, r)}{c^{r}} \frac{\Gamma(n+i)}{\Gamma(n)}\left(1-\frac{1}{c}\right)^{i} B(a+r+i, b)
\end{aligned}
$$

where $S(n, r)$ are the Stirling numbers of first kind.

\subsubsection{Increasing Factorial Moments of LNBD}

The increasing factorial moments of the LNBD is defined as:

$$
\begin{aligned}
E\left[X^{(r)}\right] & =E\left(\prod_{i=1}^{r} X+i-1\right) \\
& =\sum_{r=0}^{n} d(n, r) E\left(X^{r}\right) \\
& =\frac{1}{B(a, b)} \sum_{\mathrm{r}=0}^{n} \sum_{i=0}^{\infty} \frac{d(n, r)}{c^{r}} \frac{\Gamma(n+i)}{\Gamma(n)}\left(1-\frac{1}{c}\right)^{i} B(a+r+i, b)
\end{aligned}
$$


where $d(n, r)$ can be deduced from the relation $S(n, r)=(-1)^{n-r} d(n, r)$

2.9 Negative Moments

The negative moments of the $p d f(2.1)$ is:

$$
E\left(X^{-r}\right)=\frac{c^{r}}{B(a, b)} \sum_{i=0}^{\infty}(-1)^{i} \frac{\Gamma(n+i)}{\Gamma(n)}\left(1-\frac{1}{c}\right)^{i} B(a-r+i, b)
$$

\subsection{Incomplete Moments}

The Incomplete moments of the $p d f(2.1)$ is defined as:

$$
\varphi_{s}(t)=\frac{1}{c^{s} B(a, b)} \sum_{i=0}^{\infty} \frac{\Gamma(n+i)}{\Gamma(n)}\left(1-\frac{1}{c}\right)^{i} B_{c x /[1+(c-1) x]}(a+s+i, b)
$$

\subsection{Scaled Total Time for Aging Properties}

The scaled total time of the $p d f(2.1)$ is defined as:

$$
\begin{aligned}
& S_{F}(F(t))=\frac{1}{\mu} \int_{0}^{x} S(y) d y=\frac{1}{\mu} \int_{0}^{x} \int_{y}^{1} f(t) d t d y \\
& S_{F}(F(t))=\frac{1}{\mu}\left(x-\frac{1}{B(a, b)} \int_{0}^{x} \int_{0}^{\mathrm{cy} /[1+(\mathrm{c}-1) y]} t^{a-1}(1-t)^{b-1} d t d y\right) \\
& S_{F}(F(x))=\frac{1}{\mu}\left(x-\frac{1}{B(a, b)} \int_{0}^{x} I_{\mathrm{cy} /[1+(\mathrm{c}-1) y]}(a, b) d y\right)
\end{aligned}
$$

\subsection{Conditional Moments}

The conditional moments of the $p d f(2.1)$ is defined as:

$$
\begin{aligned}
& M_{r}^{\prime}=E\left[X^{r} \mid X>x\right] \\
& M_{r}^{\prime}=\frac{1}{\bar{F}} \int_{x}^{1} t^{r} f(t) d t
\end{aligned}
$$

By applying the substitution (2.1) and (2.2) and after some simplification, we have

$$
M_{r}^{\prime}=\frac{c^{a}\left[\mu_{r}^{\prime}-\mathrm{I}_{c x /[1+(c-1) x]}(a+r, b)\right]}{B(a, b)\left[1-I_{c x /[1+(c-1) x]}(a, b)\right]}
$$

\subsection{Mean Residual Function}

The mean residual function of the $p d f(2.1)$ is defined as: 


$$
\begin{aligned}
& m(x)=E[X-x \mid X>x] \\
& m(x)=E[X \mid X>x]-x \\
& m(x)=\frac{c^{a}\left[\mu_{1}^{\prime}-I_{c x /[1+(c-1) x]}(a+1, b)\right]}{B(a, b)\left[1-I_{c x /[1+(c-1) x]}(a, b)\right]}-x
\end{aligned}
$$

\subsection{Vitality Function}

The vitality function of the $p d f(2.1)$ is defined as:

$$
\begin{aligned}
& V(x)=E[X \mid X>x] \\
& V(x)=\frac{c^{a}\left[\mu_{1}^{\prime}-I_{c x /[1+(c-1) x]}(a+1, b)\right]}{B(a, b)\left[1-I_{c x /[1+(c-1) x]}(a, b)\right]}
\end{aligned}
$$

\subsection{Gometric Vitality Function}

The geometric vitality function of the $p d f(2.1)$ is defined as:

$$
\begin{aligned}
& \log G(x)=E[\log X \mid X>x] \\
& \log G(x)=\frac{1}{1-F(t)} \int_{x}^{1} \log t f(t) d t
\end{aligned}
$$

By using (2.1), (2.2) and (2.3), we have

$$
\log G(x)=\frac{c^{a}\left(\int_{1}^{c x /[1+(c-1) x]} \ln y y^{a-1}(1-y)^{b-1} d y-\int_{1}^{c x /[1+(c-1) x]} \ln \{c+(1-c) y\} y^{a-1}(1-y)^{b-1} d y\right)}{B(a, b)\left[1-I_{c x /[1+(c-1) x]}(a, b)\right]}
$$

\subsection{Characteristics Function}

The characteristic function of the $p d f(2.1)$ is defined as:

$$
\phi_{x}(t)=E\left(e^{i t x}\right)
$$




$$
=\frac{1}{B(a, b)} \sum_{r=0}^{\infty} \sum_{j=0}^{\infty} \frac{\Gamma(n+j)}{\Gamma(n)}\left(1-\frac{1}{c}\right)^{j} B(a+r+j, b) \frac{(j t)^{r}}{c^{r} r !}
$$

\subsection{Information generating function}

The information generating function of the (2.1) is defined by $P(s)$ and found from

$$
\begin{aligned}
P(s) & =E\left(f^{s-1}\right) \\
& =\frac{c^{s . a}}{(B(a, b))^{s}} \int_{0}^{1} \frac{t^{s a-s}(1-t)^{s b-s}}{[1-(1-c) t]^{(a+b) t}} d x
\end{aligned}
$$

By applying the substitution (2.1) and (2.2), we have

$$
P(s)=\frac{c^{s-1}}{[B(a, b)]^{s}} \sum_{i=0}^{\infty} \frac{\Gamma(2 s+i-2)}{\Gamma(2 s-2)}\left(1-\frac{1}{c}\right)^{i} B(s(a-1)+i+1, \mathrm{~s}(b-1)+1)
$$

\subsection{Points of Inflection}

The points of inflection of the $p d f(2.1)$ are found from

$$
\begin{aligned}
f^{\prime \prime}(x) & =0 \text { under } f^{\prime \prime \prime}(x) \neq 0 \text { or equivalentally as } \frac{d^{2}}{d x^{2}} \ln f(x)=0 \& \frac{d^{3}}{d x^{3}} \ln f(x) \neq 0 \\
\frac{d^{2}}{d x^{2}} \ln f(x) & =-\frac{a-1}{x^{2}}-\frac{b-1}{(1-x)^{2}}+\frac{(a+b)(1-c)^{2}}{[1-(1-c) x]^{2}}-\frac{a-1}{x^{2}}-\frac{b-1}{(1-x)^{2}}+\frac{(a+b)(1-c)^{2}}{[1-(1-c) x]^{2}}=0
\end{aligned}
$$

After some simplification

$A x^{4}+B x^{3}+C x^{2}+D x+E=0$

where

$$
\begin{aligned}
& A=2(c-1)^{2}, B=-2(c-1)(a+b c+c-3), C=(c-1)(3 a-2 b+b c+c-3) \\
& D=-2(a-1)(c-2) \text { and } E=-(a-1)
\end{aligned}
$$

It is four degree equation; it can either be solved analytically or through software for specific values of parameters. 
Table 2.3 The Points of inflection for the LNBD (For fixed values)

\begin{tabular}{cccc}
\hline $\mathrm{a}$ & $\mathrm{b}$ & $\mathrm{c}$ & Points of inflection \\
\hline 2.0 & 2 & 2 & 0.47248 \\
2.5 & 2.5 & 2.5 & 0.03203 \\
3.0 & 3.0 & 3.0 & $0.04577,0.31463$ \\
3.5 & 3.5 & 3.5 & $0.05223,0.27525$ \\
4.0 & 4.0 & 4.0 & $0.05522,0.24560$ \\
4.5 & 4.5 & 4.5 & $0.05637,0.22252$ \\
5.0 & 5.0 & 5.0 & $0.05651,0.20302$ \\
5.5 & 5.5 & 5.5 & $0.05605,0.18703$ \\
\hline
\end{tabular}

Table 2.4 The Points of inflection for the LNBD (For different values)

\begin{tabular}{cccc}
\hline $\mathrm{a}$ & $\mathrm{b}$ & $\mathrm{c}$ & Points of inflection \\
\hline 2.0 & 5.0 & 0.1 & 0.77523 \\
2.0 & 5.0 & 0.2 & $0.54776,0.89745$ \\
2.0 & 5.0 & 0.3 & $0.31849,0.83656$ \\
3.0 & 6.5 & 0.1 & $0.78934,0.94129$ \\
3.0 & 6.5 & 0.2 & $0.32553,0.60080$ \\
3.0 & 6.5 & 0.3 & $0.46964,0.44414$ \\
4.0 & 8.0 & 0.1 & $0.79880,0.93391$ \\
4.0 & 8.0 & 0.2 & $0.62896,0.86694$ \\
4.0 & 8.0 & 0.3 & $0.49366,0.80073$ \\
5.0 & 9.5 & 0.1 & $0.80560,0.92840$ \\
5.0 & 9.5 & 0.2 & $0.64689,0.85817$ \\
5.0 & 9.5 & 0.3 & $0.52260,0.79087$ \\
\hline & & & \\
\hline & & & \\
\hline & & & \\
\hline
\end{tabular}




\subsection{Reliability Measures}

The $s f$ of LNBD is denoted by $R(\mathrm{x})$ and is defined as under with graphs for various values of the parameters:

$$
\begin{aligned}
& R(x)=1-F(x) \\
& R(x)=1-I_{c x /[1+(c-1) x]}(a, b)
\end{aligned}
$$
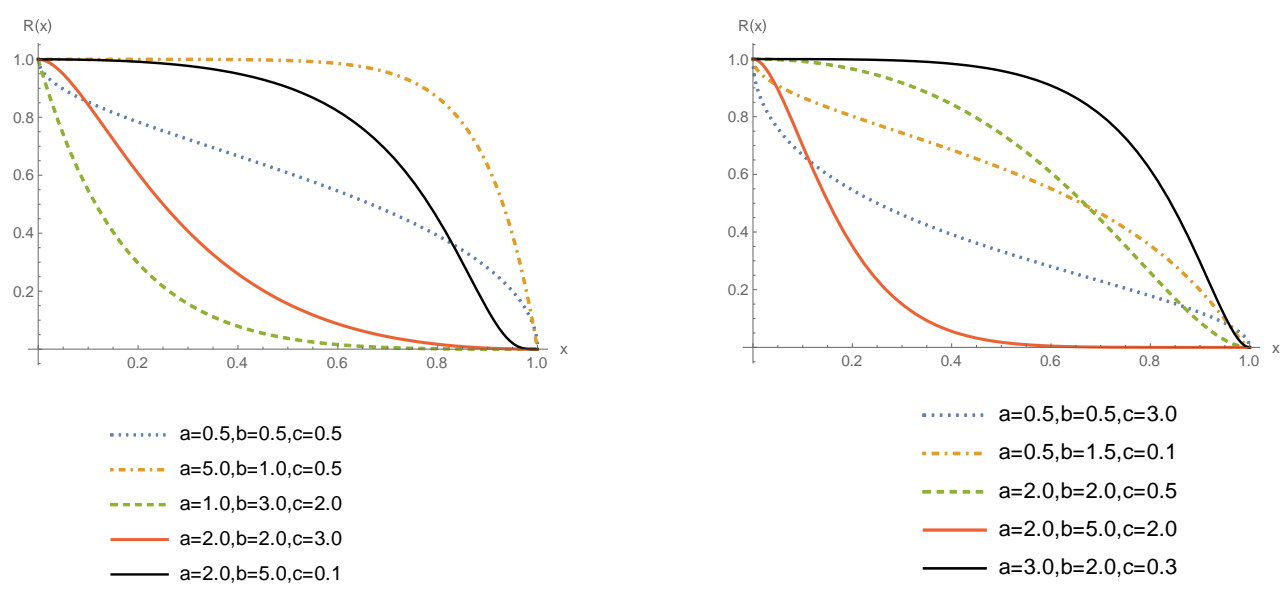

Figure 2.3

\subsection{Hazard Function (hrf)}

The hazard or instantaneous rate function is denoted by $\mathrm{H}(\mathrm{x})$. The hazard function of $\mathrm{x}$ can be interpreted as instantaneous rate or the conditional probability density of failure at time $\mathrm{x}$, given that the unit has survived until $x$. The hazard function is defined to be

$$
\begin{aligned}
& h(x)=f(x)(\bar{F}(x))^{-1} \\
& h(x)=\frac{c^{a} x^{a-1}(1-x)^{b-1}}{B(a, b)[1-(1-c) x]^{a+b}\left[1-\mathrm{I}_{c x /[1+(c-1) x]}(a, b)\right]}
\end{aligned}
$$
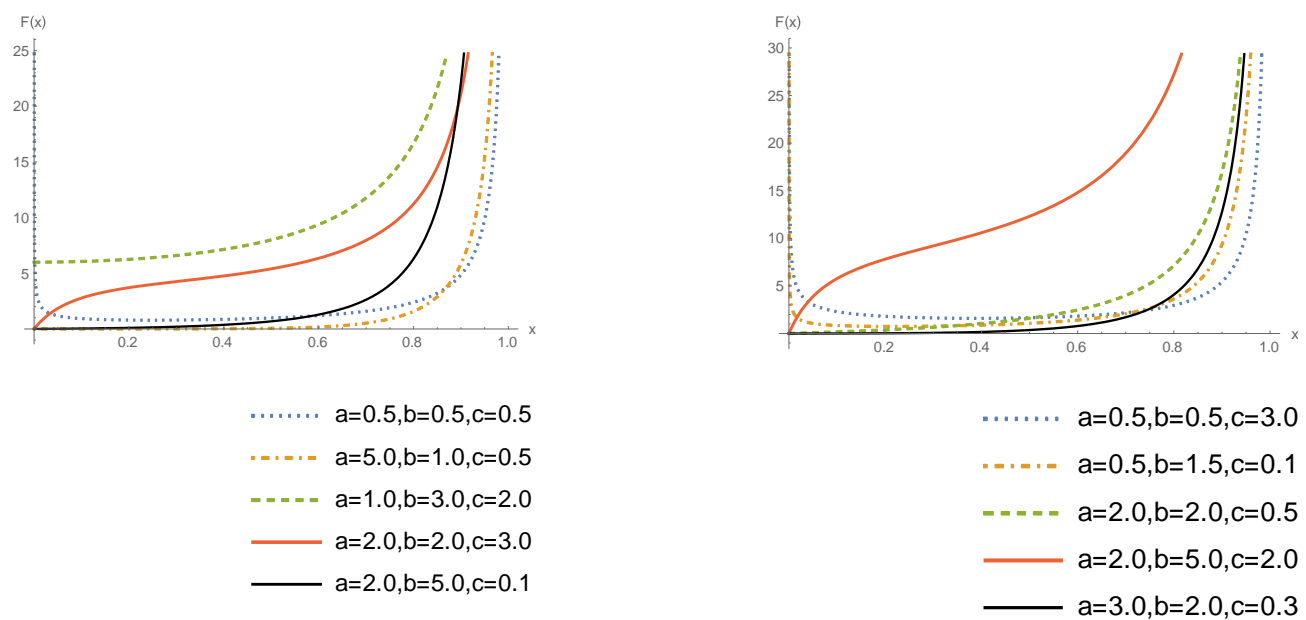

Figure 2.4 


\subsection{Reverse Hazard Function}

The reverse $h r f$ of LNBD is defined as.

$$
\begin{aligned}
& r(x)=f(x)[F(x)]^{-1} \\
& r(x)=\frac{c^{a} x^{a-1}(1-x)^{b-1}}{B(a, b)[1-(1-c) x]^{a+b} \mathrm{I}_{c x /[1+(c-1) x]}(a, b)}
\end{aligned}
$$

\subsection{Bonferroni Curve}

The Bonferroni curve of the $p d f(2.1)$ is defined as:

$$
\begin{aligned}
B_{F}(F(x)) & =\frac{1}{\mu F(x)} \int_{0}^{x} y f(y) d y \\
B_{F}(F(x)) & =\frac{\sum_{i=0}^{\infty} \frac{\Gamma(n+i)}{\Gamma(n)}\left(1-\frac{1}{c}\right)^{i} B_{c x /[1+(c-1) x]}(a+1+i, b)}{I_{c x /[1+(c-1) x]}(a, b) \sum_{i=0}^{\infty} \frac{\Gamma(n+i)}{\Gamma(n)}\left(1-\frac{1}{c}\right)^{i} B(a+r+i, b)}
\end{aligned}
$$

\subsection{Lorenz Curve}

The Lorenz curve of the $p d f(2.1)$ is defined as:

$$
\begin{aligned}
& L(F(x))=F(x) B(F(x)) \\
& L(F(x))=\frac{\sum_{i=0}^{\infty} \frac{\Gamma(n+i)}{\Gamma(n)}\left(1-\frac{1}{c}\right)^{i} B_{c x /[1+(c-1) x]}(a+1+i, b)}{\sum_{i=0}^{\infty} \frac{\Gamma(n+i)}{\Gamma(n)}\left(1-\frac{1}{c}\right)^{i} B(a+r+i, b)}
\end{aligned}
$$

\subsection{Gini Coefficient}

The Gini coefficient of the $p d f(2.1)$ is explained as:

$$
\begin{aligned}
& G=1-\frac{1}{\mu} \int_{0}^{1}(1-F(x))^{2} d x \\
& =1-\frac{1}{\mu} \int_{0}^{1}\left[1-\frac{1}{B(a, b)} \int_{0}^{c x /[1+(c-1) x]} t^{a-1}(1-t)^{b-1} d t\right]^{2} d x
\end{aligned}
$$


Table 2.6 The Gini Coefficient for the LNBD

\begin{tabular}{cccc}
\hline $\mathrm{a}$ & $\mathrm{b}$ & $\mathrm{c}$ & Gini Coefficient \\
\hline 2 & 2 & 2 & 0 \\
2.5 & 2.5 & 2.5 & 0.13001 \\
3 & 3 & 3 & 0.11912 \\
4 & 4 & 4 & 0.10052 \\
4.5 & 4.5 & 4.5 & 0.09282 \\
5 & 5 & 5 & 0.08602 \\
5.5 & 5.5 & 5.5 & 0.08003 \\
\hline
\end{tabular}

Table 2.7 The Gini Coefficient for the LNBD

\begin{tabular}{cccc}
\hline $\mathrm{a}$ & $\mathrm{b}$ & $\mathrm{c}$ & Gini Coefficient \\
\hline 0.5 & 0.5 & 3.0 & 0.37516 \\
0.5 & 1.0 & 2.5 & 0.39541 \\
0.5 & 1.5 & 2.0 & 0.22469 \\
0.5 & 1.0 & 0.5 & 0.16378 \\
1.0 & 2.0 & 3.0 & 0.28570 \\
2.0 & 3.0 & 4.0 & 0.03762 \\
3.0 & 4.0 & 5.0 & 0.10899 \\
4.0 & 5.0 & 6.0 & 0.11417 \\
5.0 & 6.0 & 0.7 & 0.10840 \\
6.0 & 0.7 & 0.8 & 1.10059 \\
\hline
\end{tabular}

\subsection{Asymptotes and Shapes}

The asymptotes of (1.1), (1.2) and (3.1) as $x \rightarrow 0,1$ are as explained below: 
Table 2.8

\begin{tabular}{lccc}
\hline & $p d f f(x)$ & $c d f F(x)$ & $h r f h(x)$ \\
\hline$x \rightarrow 0$ & $\frac{c^{a} x^{a-1}}{B(a, b)}$ & $\frac{c^{a} x^{a}}{a B(a, b)}$ & $\frac{c^{a} x^{a-1}}{B(a, b)}$ \\
& $\frac{(1-x)^{b-1}}{c^{b} B(a, b)}$ & $1-\frac{c^{a}(1-x)^{b}}{b c^{b} B(a, b)}$ & $\frac{b}{1-x}$ \\
\hline
\end{tabular}

\subsection{Transformation}

Table 2.8 Different transformation of LNBD

\begin{tabular}{|c|c|c|c|c|c|}
\hline Sr. No. & $a$ & $\boldsymbol{b}$ & $c$ & Transformation & Resulting Distribution \\
\hline 1 & $a$ & $b$ & 1 & $x$ & Beta Type - I $B(a, b)$ \\
\hline 2 & $a$ & $b$ & 1 & $1-x$ & Miror of Beta $B(a, b)$ \\
\hline 3 & $a$ & $b$ & 1 & $\frac{x}{1-x}$ & Beta $2^{\text {nd }}$ kind or $B^{\prime}(a, b)$ \\
\hline 4 & $a$ & $b$ & $c$ & $\frac{c x}{1-x}$ & $B^{\prime}(a, b)$ \\
\hline 5 & $\frac{n}{2}$ & $\frac{m}{2}$ & 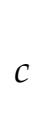 & $\frac{m c x}{n(1-x)}$ & $F(n, m)$ \\
\hline 6 & 1 & $b$ & 1 & $x^{\alpha}$ & $\operatorname{Kum}(\alpha, b)$ \\
\hline 7 & 1 & $b$ & 1 & $x$ & $\operatorname{Kum}(1, b)$ \\
\hline 8 & $a$ & 1 & 1 & $-\ln x$ & $\exp (a)$ \\
\hline 9 & $a$ & $b$ & $c$ & $\frac{c x}{1-(1-c) x}$ & Beta Type - I $\mathrm{B}(a, b)$ \\
\hline 10 & 1 & $b$ & C & $\frac{c x^{\alpha}}{1-(1-c) x^{\alpha}}$ & Libby-Novick $(b, c, \alpha)$ \\
\hline 11 & 1 & 1 & 1 & $x$ & Uniform $U(0,1)$ \\
\hline 12 & $\frac{3}{2}$ & $\frac{3}{2}$ & 1 & $2 r x-r$ & Wigner sanecar distribution \\
\hline 13 & $\frac{1}{2}$ & $\frac{1}{2}$ & 1 & $x$ & Arcsine distribution \\
\hline
\end{tabular}




\section{CHARACTERIZATION}

\subsection{Based on one truncated moment}

From [32], [33] the following two lemmas to characterize different univariate continuous distributions. Here, we discuss characterizations of $L N B G$ class distributions through one truncated moment.

\section{Assumption 3.1}

Suppose the random variable $\mathrm{X}$ is absolutely continuous with cumulative distribution function (cdf) $\mathrm{F}(\mathrm{x})$ and probability density function (pdf) $\mathrm{f}(\mathrm{x})$. We assume that $\gamma=\sup \{x \mid F(x)>0\}$ and $\delta=\inf \{x \mid F(x)<1\}$. We further assume that $\mathrm{E}(\mathrm{X})$ exists.

Lemma 3.1 Under the Assumption 3.1 if $E(X \mid X \leq x)=g(x) \tau(x)$, where $\tau(x)=\frac{f(x)}{F(x)}$ and $g(x)$ is a continuous differentiable function of $x$ with the condition that $\int_{\gamma}^{x} \frac{u-g^{\prime}(u)}{g(u)} d u$ is finite for all $x, \gamma \leq x \leq \delta$, and then $f(x)=c e^{\int_{\gamma}^{x} \frac{u-g^{\prime}(u)}{g(u)} d u}$, where $c$ is determined by the condition $\int_{\gamma}^{\delta} f(x) d x=1$

Proof. It follows from Ahsanullah et al., (2016) and Ahsanullah (2017).

Lemma 3.2 Under the Assumption 3.1, if $E(X \mid X \geq x)=h(x) r(x)$, where $r(x)=\frac{f(x)}{1-F(x)}$ and $h(x)$ is a continuous differentiable function of $x$ with the condition that $\int_{\gamma}^{x} \frac{u+h^{\prime}(u)}{h(u)} d u$ is finite for all $x, \gamma \leq x \leq \delta$, then $f(x)=c e^{-\int_{\gamma}^{x} \frac{u+h^{\prime}(u)}{h(u)} d u}$, where $\mathrm{c}$ is determined by the condition $\int_{\gamma}^{\delta} f(x) d x=1$

Proof. It follows from [32], [33]. 


\section{Theorem 3.2}

Under the assumption 3.1 with $\gamma=0$ and $\delta=1$, if $E(X \mid X \leq x)=g(x) \tau(x)$, where

$g(x)=\frac{B(a, b)[1-(1-c) x]^{a+b}}{c^{a} x^{a-1}(1-x)^{b-1}} M_{1}^{\prime}$ and $\tau(x)=\frac{f(x)}{F(x)}$, if and only if

$f(x)=\frac{c^{a} x^{a-1}(1-x)^{b-1}}{B(a, b)[1-(1-c) x]^{a+b}}$

Proof.

If $f(x)=\frac{c^{a} x^{a-1}(1-x)^{b-1}}{B(a, b)[1-(1-c) x]^{a+b}}$, then $f(x) g(x)=\int_{0}^{x} u f(u) d u=M_{1}^{\prime}$

Thus

$g(x)=\frac{B(a, b)[1-(1-c) x]^{a+b}}{c^{a} x^{a-1}(1-x)^{b-1}} M_{1}^{\prime}$

Suppose $g(x)=\frac{B(a, b)[1-(1-c) x]^{a+b}}{c^{a} x^{a-1}(1-x)^{b-1}} M_{1}^{\prime}$, then

$g^{\prime}(x)=x+\frac{B(a, b)[1-(1-c) x]^{a+b}}{c^{a} x^{a-1}(1-x)^{b-1}} M_{1}^{\prime}\left[-\frac{(1-c)(a+b)}{1-(1-c) x}-\frac{a-1}{x}+\frac{b-1}{1-x}\right]$

$g^{\prime}(x)=x+g(x)\left[-\frac{(1-c)(a+b)}{1-(1-c) x}-\frac{a-1}{x}+\frac{b-1}{1-x}\right]$

Thus

$$
\frac{x-g^{\prime}(x)}{g(x)}=\frac{(1-c)(a+b)}{1-(1-c) x}+\frac{a-1}{x}-\frac{b-1}{1-x}
$$

By Lemma 3.1 and after some simplification, we obtain the following result $f(x)=k[1-(1-c) x]^{-(a+b)} x^{a-1}(1-x)^{b-1}$, where $k$ is a constant. 
Using the condition $\int_{0}^{1} f(x) d x=1$, we obtain

$$
f(x)=\frac{c^{a}}{B(a, b)} \frac{x^{a-1}(1-x)^{b-1}}{[1-(1-c) x]^{a+b}}
$$

\section{Theorem 3.3}

Under the assumption 3.1 with $\gamma=0$ and $\delta=1$, if $\mathrm{E}(\mathrm{X} \mid \mathrm{X} \geq x)=\mathrm{h}(x) \mathrm{r}(x)$, where

$$
\begin{aligned}
& h(x)=\frac{B(a, b)[1-(1-c) x]^{a+b}}{c^{a} x^{a-1}(1-x)^{b-1}}\left(E(X)-M_{1}^{\prime}\right) \text { and } r(x)=\frac{f(x)}{1-F(x)}, \text { if and only if } \\
& f(x)=\frac{c^{a}}{B(a, b)} \frac{x^{a-1}(1-x)^{b-1}}{[1-(1-c) x]^{a+b}}
\end{aligned}
$$

Proof.

If $f(x)=\frac{c^{a}}{B(a, b)} \frac{x^{a-1}(1-x)^{b-1}}{[1-(1-c) x]^{a+b}}$, then $f(x) h(x)=\int_{x}^{1} u f(u) d u=E(X)-M_{1}^{\prime}$

Thus

$$
h(x)=\frac{B(a, b)[1-(1-c) x]^{a+b}}{c^{a} x^{a-1}(1-x)^{b-1}}\left(E(X)-M_{1}^{\prime}\right)
$$

Suppose

$$
\begin{aligned}
& h(x)=\frac{B(a, b)[1-(1-c) x]^{a+b}}{c^{a} x^{a-1}(1-x)^{b-1}}\left(E(X)-M_{1}^{\prime}\right), \text { then } \\
& h^{\prime}(x)=-x+\frac{B(a, b)[1-(1-c) x]^{a+b}}{c^{a} x^{a-1}(1-x)^{b-1}}\left(E(X)-M_{1}^{\prime}\right)\left[-\frac{(1-c)(a+b)}{1-(1-c) x}-\frac{a-1}{x}+\frac{b-1}{1-x}\right] \\
& h^{\prime}(x)=-x+h(x)\left[-\frac{(1-c)(a+b)}{1-(1-c) x}-\frac{a-1}{x}+\frac{b-1}{1-x}\right]
\end{aligned}
$$




$$
\frac{-x+h^{\prime}(x)}{h(x)}=\frac{(1-c)(a+b)}{1-(1-c) x}+\frac{a-1}{x}-\frac{b-1}{1-x}
$$

By Lemma 3.2

$$
\frac{f^{\prime}(x)}{f(x)}=\frac{(1-c)(a+b)}{1-(1-c) x}+\frac{a-1}{x}-\frac{b-1}{1-x}
$$

Integrating the both sides of the above equation with respect to $x$, we obtain

$f(x)=k \frac{x^{a-1}(1-x)^{b-1}}{[1-(1-c) x]^{a+b}}$, where $k$ is a constant.

Using the condition $\int_{0}^{1} f(x) d x=1$, we obtain

$$
f(x)=\frac{c^{a}}{B(a, b)} \frac{x^{a-1}(1-x)^{b-1}}{[1-(1-c) x]^{a+b}}
$$

\subsection{Maximum-likelihood estimation}

\section{ESTIMATION}

Let $x_{1}, x_{2}, x_{3}, \ldots, x_{n}$ be a random sample having probability density function

$$
\begin{aligned}
& L(x ; a, b, c)=\prod_{i=1}^{n} f(x) \\
& L(x ; a, b, c)=\frac{c^{n a}}{[B(a, b)]^{n}} \frac{\left(\prod_{i=1}^{n} x_{i}\right)^{a-1}\left(\prod_{i=1}^{n}(1-x)\right)^{b-1}}{\left[\prod_{i=1}^{n}\left\{1-(1-c) x_{i}\right\}\right]^{a+b}}
\end{aligned}
$$

The log-likelihood function is

$$
\begin{aligned}
\ln L(x ; a, b, c)= & n a \ln c-n \ln B(a, b)+(a-1) \sum_{i=1}^{n} \ln x_{i}+(b-1) \sum_{i=1}^{n} \ln \left(1-x_{i}\right) \\
& -(a+b) \sum_{i=1}^{n} \ln \left[1-(1-c) x_{i}\right]
\end{aligned}
$$

It follows that the maximum-likelihood estimates $(a, b, c)$, say $($ 次 $\hat{b}, c)$, are the simultaneous solutions of the equations: 


$$
\begin{aligned}
& 0=\frac{\partial \ln L}{\partial \mathbf{a}}=n \ln c-n[\psi(a)-\psi(a+b)]+\sum_{i=1}^{n} \ln x_{i}-\sum_{i=1}^{n} \ln \left[1-(1-c) x_{i}\right] \\
& 0=\frac{\partial \ln L}{\partial b}=-n[\psi(b)-\psi(a+b)]+\sum_{i=1}^{n} \ln \left(1-x_{i}\right)-\sum_{i=1}^{n} \ln \left[1-(1-c) x_{i}\right] \\
& 0=\frac{\partial \ln L}{\partial c}=\frac{a n}{c}-(a+b) \sum_{i=1}^{n} \frac{c}{1-(1-c) x_{i}}
\end{aligned}
$$

These equations can be easily solved by using the R statistical package.

\subsection{A simulation study}

The study was based on 1000 generated data sets from the LNBD with different values of the parameters for $n=10,20,30,50,70,100,150,200,300,500,700$ and 1000. We calculate of the bias and MSE for different values of the parameters, these results suggest that the MSE decrease when sample size increases.

Table 4.2.1 The Bias, MSE values for the LNB model when $a=b=c=2.5$

\begin{tabular}{ccccccc}
\hline & $\mathbf{n}=\mathbf{1 0}$ & $\mathbf{n}=\mathbf{2 0}$ & $\mathbf{n}=\mathbf{3 0}$ & $\mathbf{n}=\mathbf{5 0}$ & $\mathbf{n}=\mathbf{7 0}$ & $\mathbf{n}=\mathbf{1 0 0}$ \\
\hline Bias (a) & -2.05042 & -2.49065 & -0.7122865 & -0.86810 & -0.58077 & 0.57136 \\
Bias (b) & -1.50497 & -2.01256 & -2.171628 & 15.99567 & 17.03842 & 27.10133 \\
Bias (c) & -2.40205 & -2.071787 & -2.466312 & 0.177780 & -1.71462 & -1.41986 \\
\hline MSE (a) & 67.98561 & 64.23299 & 55.0937 & 41.68756 & 9.82927 & 1.43641 \\
MSE (b) & 258.9330 & 237.71409 & 195.15059 & 189.3684 & 149.6461 & 111.326 \\
MSE (c) & 65.35506 & 56.42674 & 47.268447 & 36.1156 & 30.09384 & 19.86549 \\
\hline & $\mathbf{n}=\mathbf{1 5 0}$ & $\mathbf{n}=\mathbf{2 0 0}$ & $\mathbf{n = 3 0 0}$ & $\mathbf{n}=500$ & $\mathbf{n = 7 0 0}$ & $\mathbf{n}=\mathbf{1 0 0 0}$ \\
\hline Bias (a) & 0.45077 & 0.38837 & 0.36992 & -1.64893 & 0.30763 & 0.30824 \\
Bias (b) & 21.20987 & 15.69702 & 13.10631 & 9.31559 & 8.41728 & 7.78502 \\
Bias (c) & -1.67034 & -1.714691 & -1.76891 & -1.76459 & -1.79250 & 0.30603 \\
\hline MSE (a) & 0.91711 & 0.51585 & 0.34143 & 4.45167 & 0.17203 & 0.15320 \\
MSE (b) & 1411.456 & 728.7420 & 459.8135 & 164.5743 & 107.269 & 81.3317 \\
MSE (c) & 3.49029 & 3.31447 & 3.30728 & 3.233171 & 3.30078 & 0.14827 \\
\hline
\end{tabular}

5 Application

In order to prove that LNBD can be a better model than the Power distribution, Beta distribution with $(\mathrm{a}=1)$, Beta distribution, Kumaraswamy distribution, let us use three real data sets.

The following tables show the numerical values with MLEs and their corresponding standard errors (in parentheses) of the model parameters including loglikelihood, Kolmogorov- 
Smirnov test (KS), Akaike information criterion (AIC) and Consistent Akaike information criterion (CAIC) for comparing LNBD with the Power distribution, Beta distribution with $(\mathrm{a}=$ 1), Beta distribution, Kumaraswamy distribution. It is quite evident from the reports that LNBD is better. The plots of the fitted distributions to real datasets are shown in figures.

\section{Data Set 1:}

The following dataset which is skewed to right, present the SAR image modeling on oil slick visibility in ocean. The values are:

$0.6244,0.1868,0.5444,0.3399,0.4864,0.4825,0.2528,0.2612,0.2086,0.3303,0.5453,0.2025,0.4231$, $0.2310,0.7167,0.2706,0.1922,0.5390,0.5550,0.2282,0.5434,0.4799,0.4570,0.3448,0.2271,0.4731$, $0.1875,0.3188,0.1824,0.3229,0.1962,0.3743,0.1614,0.1543,0.4985,0.1515,0.2553,0.1734,0.1617$, $0.2271,0.2253,0.2635,0.5441,0.1281,0.4764,0.3443,0.3770,0.5101,0.3143,0.1645,0.1211,0.2900$, $0.4265,0.2084,0.5753,0.2526,0.2469,0.2301,0.5180,0.4176,0.1776,0.6351,0.3362,0.2355,0.3916$, $0.4615,0.6178,0.3272,0.3876,0.2010,0.3614,0.1480,0.3105,0.1710,0.2771,0.4655,0.1468,0.2113$, $0.3071,0.4291,0.2777,0.2101,0.4991,0.2567,0.3065,0.5470,0.3353,0.1948,0.2686,0.2061,0.5123$, $0.1567,0.3749,0.3714,0.3618,0.5189,0.3500,0.2633,0.1928,0.4022,0.1120,0.3621,0.4664,0.3106$, $0.2465,0.2388,0.4497,0.2979,0.1524,0.1822,0.3955,0.1744,0.3800,0.4578,0.1872,0.2587,0.4699$, $0.2329,0.3943,0.3613$.

Table 5.1: Estimated Parameters by MLE with their S.E. and Goodness of Fit

\begin{tabular}{|l|c|c|c|c|c|c|c|}
\hline Model & $\hat{a}$ & $\hat{b}$ & $\hat{c}$ & $\ln (\mathbf{L})$ & KS & AIC & CAIC \\
\hline Power & $\begin{array}{c}5.7155 \times 10^{-3} \\
0.07560\end{array}$ & & & -2.2764 & 0.3442 & 6.5527 & 6.5867 \\
\hline Beta1 & & $\left.\begin{array}{c}0.04690 \\
(0.2166)\end{array}\right)$ & & -34.2384 & & 70.4768 & 70.5107 \\
\hline Beta & $\begin{array}{c}0.2118 \\
(0.4602)\end{array}$ & $\begin{array}{c}0.9438 \\
(0.9715)\end{array}$ & & -73.6054 & 0.08153 & 151.2108 & 151.3134 \\
\hline Kumaraswamy & $\begin{array}{c}4.1328 \\
(2.3293)\end{array}$ & $\begin{array}{c}0.03734 \\
(0.1932)\end{array}$ & & -71.5770 & 0.08800 & 147.1539 & 147.2565 \\
\hline Libby-Novick & $\begin{array}{c}324.8720 \\
(18.1072)\end{array}$ & $\begin{array}{c}1.6564 \\
(1.2870)\end{array}$ & $\begin{array}{c}338.7979 \\
(18.4065)\end{array}$ & -75.00942 & 0.06152 & 156.0188 & 156.2257 \\
\hline
\end{tabular}



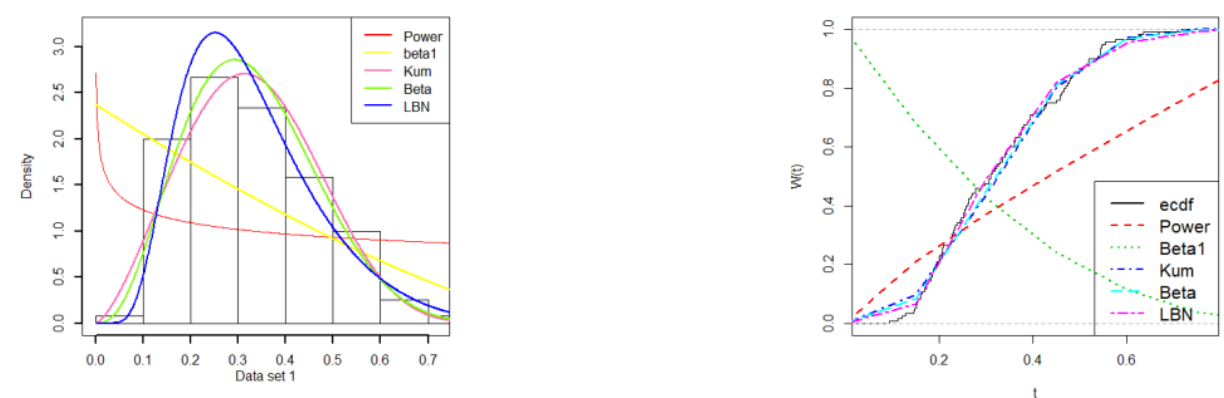

Figure 5.1: $p d f$ and cdf graphs of the densities
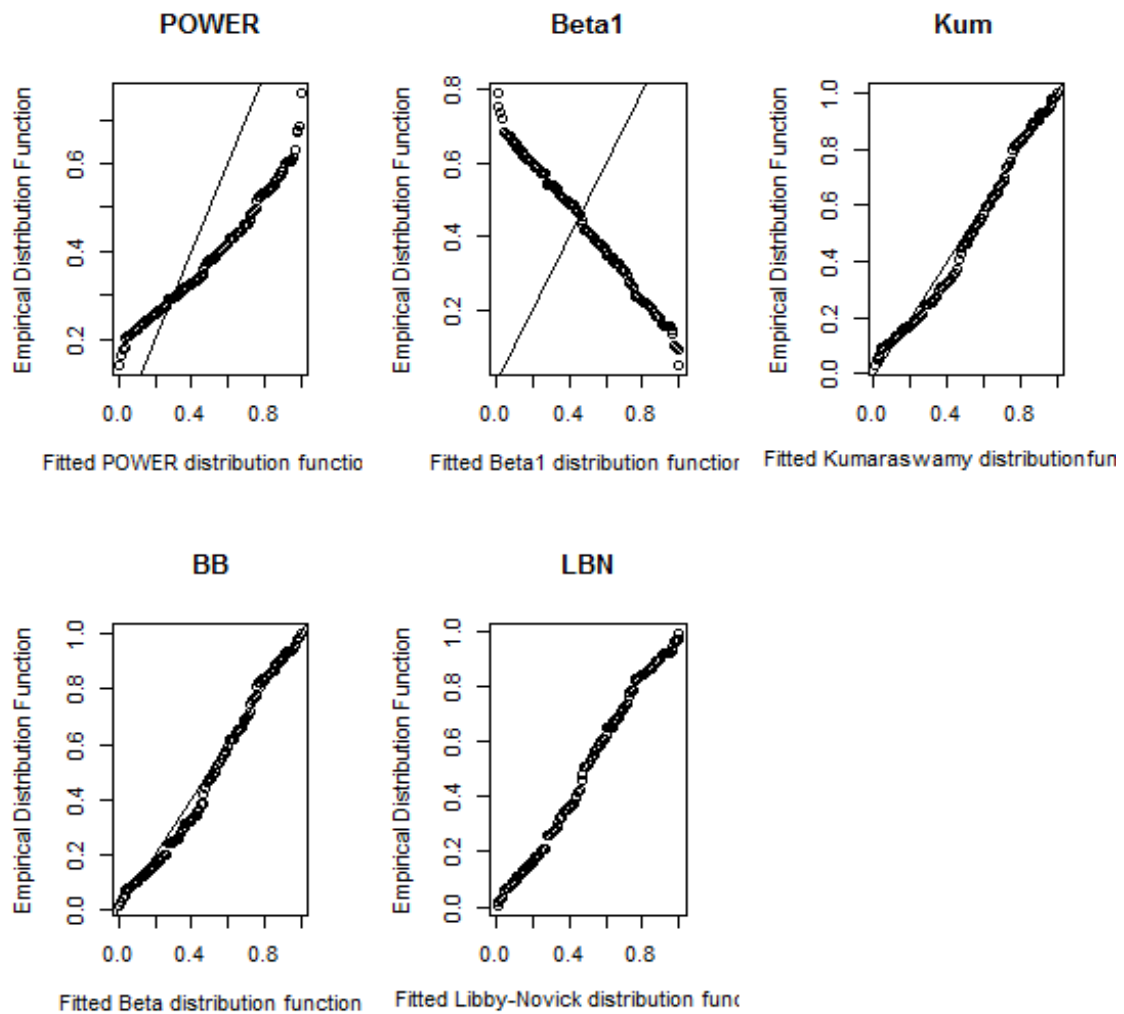

Figure 5.2: $Q-Q$ plots of the densities

\section{Data Set 2:}

The following right to skewed dataset presented by Cordeiro and Brito (2012) is obtained from the measurements on petroleum rock samples. The data consists of 48 rock samples from a petroleum reservoir. The dataset corresponds to twelve core samples from petroleum reservoirs that were sampled by four cross-sections. Each core sample was measured for permeability and 
each cross-section has the following variables: the total area of pores, the total perimeter of pores and shape. We analyze the shape perimeter by squared (area) variable and the observations are:

$0.090330,0.203654,0.204314,0.280887,0.197653,0.328641,0.448622,0.562394,0.462727,0.279455$, $0.520635,0.830081,0.183312,0.350944,0.201071,0.191802,0.054192,0.564125,0.217063,0.048141$, 0.544810, 0.433083, 0.176016, 0.420477, 0.422417, 0.228595, 0.113852, 0.025214, 0.376969, 0.200744, $0.156705,0.031623,0.791029,0.041273,0.238712,0.362651,0.389051,0.472567,0.340077,0.711646$, $0.463586,0.282453,0.264127,0.151181,0.461865,0.306016,0.553832,0.105447$

Table 5.2: Estimated Parameters by MLE with their S.E. and Goodness of Fit

\begin{tabular}{|l|c|c|c|c|c|c|c|}
\hline Model & $\hat{a}$ & $\hat{b}$ & $\hat{c}$ & $\ln (\mathbf{L})$ & KS & AIC & CAIC \\
\hline Power & $\begin{array}{c}0.01068 \\
(0.1033)\end{array}$ & & & -3.008295 & 0.2737 & 8.01659 & 8.1035 \\
\hline Beta1 & & $\begin{array}{c}0.1086 \\
(0.32948)\end{array}$ & & -12.6421 & 0.9825 & 27.2843 & 27.3712 \\
\hline Beta & $\begin{array}{c}0.08118 \\
(0.2849)\end{array}$ & $\begin{array}{c}0.4367 \\
(0.6608)\end{array}$ & & -14.8539 & 0.06485 & 33.7077 & 33.9744 \\
\hline Kumaraswamy & $\begin{array}{c}0.6939 \\
(0.8330)\end{array}$ & $\begin{array}{c}0.04087 \\
(0.2022)\end{array}$ & & -14.8116 & 0.06492 & 33.6232 & 33.8899 \\
\hline Libby-Novick & $\begin{array}{c}0.2447 \\
(0.4947)\end{array}$ & $\begin{array}{c}2.5122 \\
(1.5850)\end{array}$ & $\begin{array}{c}1.2131 \\
(1.1014)\end{array}$ & -14.8696 & 0.06488 & 35.7393 & 36.2847 \\
\hline
\end{tabular}
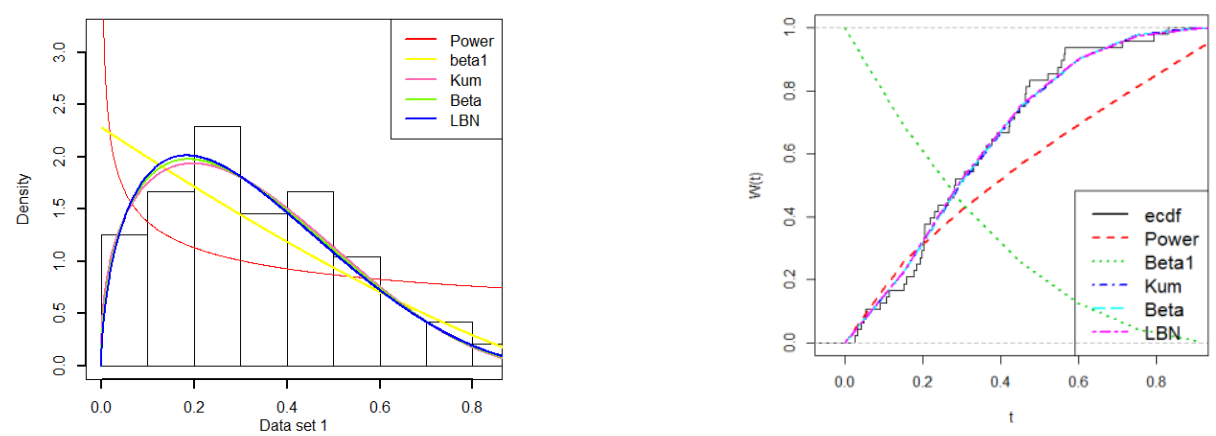

Figure 5.3: pdf and cdf graphs of the densities 

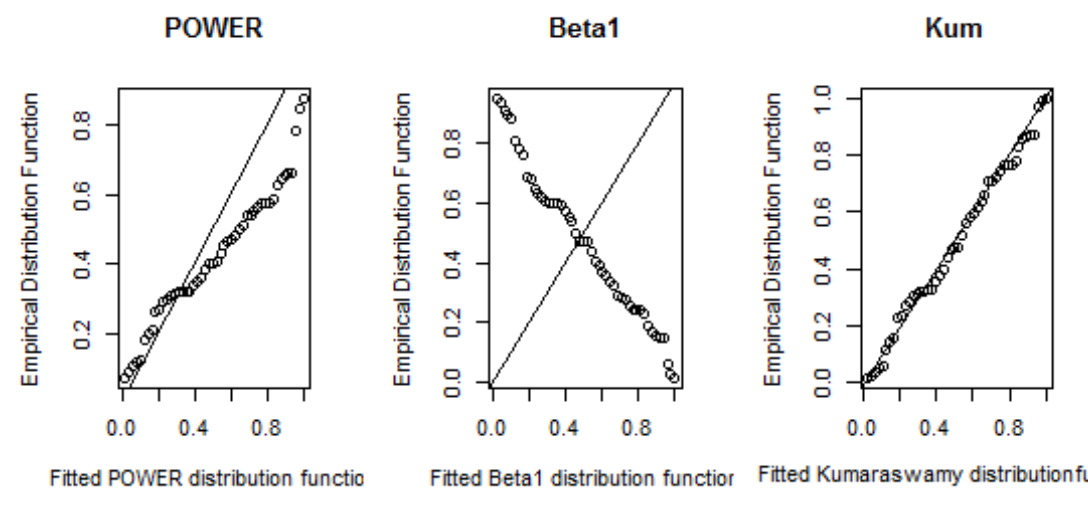

Fitted Beta1 distribution functior
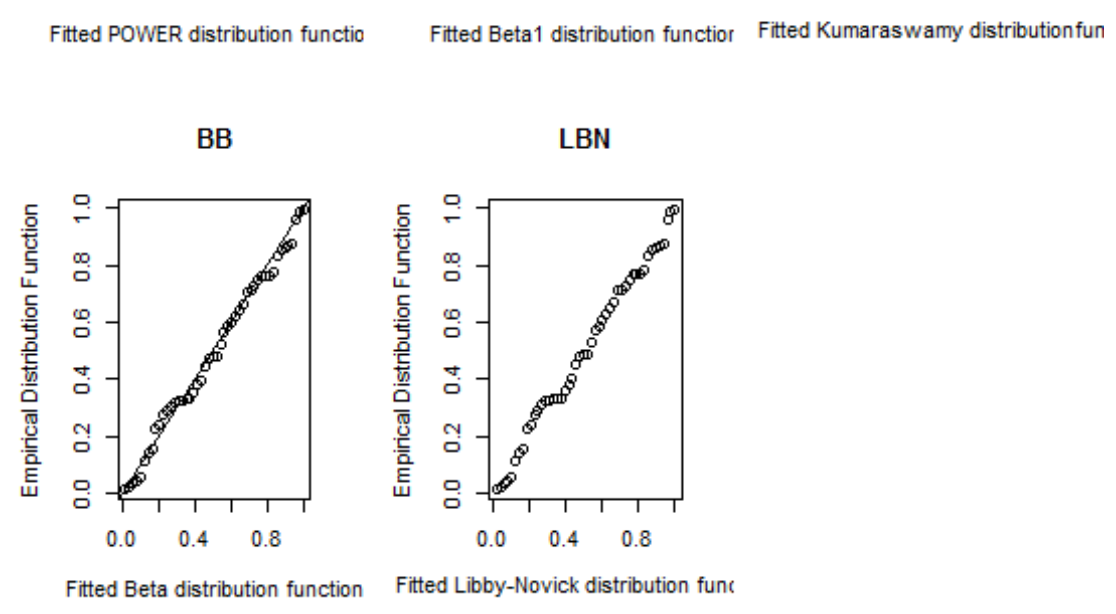

Figure 5.4: $Q-Q$ plots of the densities

\section{CONCLUSION}

This particular research work consists of basic mathematical properties of LNBD i.e. definition of probability density function ( $p d f)$ with its shape discussion, cumulative distribution function ( $c d f)$, quantiles, skewness, kurtosis, variance, mode and points of inflection along with their numerical behavior given in the form of tables due to various values of the parameters, harmonic mean, geometric mean, $\mathrm{r}^{\text {th }}$ moment about origin, moment generating function $(m g f)$, factorial moments (increasing and decreasing), negative moments, conditional moments, mean deviations from mean and median etc.

We have deduced the reliability measures in the shape of survival function, hazard rate function, reverse hazard rate function with graphical presentation. We developed transformations of LNBD to other density function, asymptotes and shapes of $p d f, c d f$ and $h r f$.

We cultivated a mathematical treatment Bonferroni and Lorenz curves, Gini Index, entropy measures, characterizations for LNBD with one truncated moment. 
We derive MLEs of the parameters, simulation study of LNBD with the comparison of Biases and MSEs due to different values of the parameters.

By means of application of LNBD to two real data sets and comparing it with the Power distribution, Beta distribution with $(\mathrm{a}=1)$, Beta distribution, Kumaraswamy distribution model, we come to the conclusion that LNBD is better.

Conflicts of Interest: The author(s) declare that there are no conflicts of interest regarding the publication of this paper.

\section{REFERENCES}

[1] A. Azzalini, A Class of Distributions Which Includes the Normal Ones, Scand. J. Stat. 12(2) (1985), 171-178.

[2] A. K. Sheikh, M. Ahmad, Z. Ali, Some remarks on the hazard functions of the inverted distributions, Reliab. Eng. 19 (1987), 255-261.

[3] A. P. Prudnikov, Y. A. Brychkov, and O.I. Marichev, Integrals and series. Vol. 1. Gordon and Breach Science Publishers, Amsterdam, (1986).

[4] A. Réyni, Probability Theory, Dover Publications, New York, 1970.

[5] A. Telcs, W. Glanzel, and A. Schubert, Characterization and Statistical Test using Truncated Expectations for a Class of Skew Distributions. Math. Soc. Sci. 10 (1985), 169-178.

[6] A. W Marshall, and I. Olkin, A new method for adding a parameter to a family of distributions with application to the exponential and weibull families. Biometrika. 84 (1997), 641-652.

[7] B.D. Sharma, I.J. Taneja, Entropy of type $(\alpha, \beta)$ and other generalized measures in information theory, Metrika. 22 (1975), 205-215.

[8] B. D. Sharma, P. Mittal, New non-additive measure of relative information. J. Comb. Inform. Syst. Sci. 2 (1977), 122-133.

[9] C. Alexander, G. M. Cordeiro, E. M. M. Ortega, J. M. Sarabia, Generalized beta-generated distributions, Comput. Stati. Data Anal. 56 (2012), 1880-1897.

[10] C.-D. Lai, G. Jones, Beta Hazard Rate Distribution and Applications, IEEE Trans. Rel. 64 (2015), 4450.

[11] C. E. Shannon, A mathematical theory of communication, SIGMOBILE Mob. Comput. Commun. Rev. 5 (2001), 3-55.

[12] C. Tsallis, Possible generalization of Boltzmann-Gibbs statistics, J. Stat. Phys. 52 (1988), 479-487.

[13] D. Chotikapanich, Modeling Income Distributions and Lorenz Curves, Springer, NY, 2008. 
[14] D. Desai, V. Mariappan, and M. Sakhardanda, Nature of Reverse Hazard Rate: An Investigation. Int. J. Perform. Eng. 7(2) (2011), 165-171.

[15] D. L. Libby, M. R. Novick, Multivariate Generalized Beta Distributions with Applications to Utility Assessment, J. Educ. Stat. 7 (1982), 271-294.

[16] D. Salomon, Data Compression, Springer, New York, 1998.

[17] D. N. Shanbagh, The Characterization for Exponential and Geometric Distributions. J. Amer. Stat. Assoc. 65(331) (1970), 1256-1259.

[18] S. T. Dara, Recent advances in moment distributions and their hazard rate. Ph.D. thesis, National College of Business Administration and Economics, Lahore, Pakistan, (2012).

[19] E. Boekee, and A. C. J. Van Der Lubbe, The R-norm information measure. Inform. Control. 45 (1980), 136-155.

[20] E. R. Barlow, W. A. Marshall, and F. Proschan, Properties of Probability Distribution with Monotone Hazard Rate. Ann. Math. Stat. 34(2) (1963), 375-389.

[21] E. M. Ghitany, The Monotonicity of the Reliability Measures of the Beta Distribution. Appl. Math. Lett. 17 (2004), 1277-1283.

[22] F. Farmoye, C. Lee and O. Olumolade, The beta-weibull distribution. J. Stat. Theory Appl. 4 (2005), 121-136.

[23] G. M. Cordeiro, L. H. de Santana, E. M. M. Ortega, R. R. Pescim, A New Family of Distributions: Libby-Novick Beta, Int. J. Stat. Probab. 3 (2014), 63-80.

[24] G. G. Hamedani, On certain generalized gamma convolution distributions II. Technical Report, No. 484, MSCS, Marquette University, 2013.

[25] G. M. Cordeiro, M. de Castro, A new family of generalized distributions. J. Stat. Comput. Simul. 81(7) (2011), 883-898.

[26] A. K. Gupta, S. Nadarajah, Handbook of beta distribution and its applications. Marcel Dekker, New York, 2004.

[27] I. S. Gradshteyn, I. M. Ryzhik, Table of integrals, series and products. 7th ed. Academic Press, Diego, 2004.

[28] J. Havrda, F. S. Charvat, Quantification method of classification processes: Concept of structuralentropy, Khbernetika. 3(1967), 30-35.

[29] J. Navarro, A. Guillamon, M.C. Ruiz, Generalized mixture in reliability modeling: Applications to the construction of bathtub shaped hazard model and the study of systems. Appl. Stoch. Models Bus. Ind. 25(3) (2009), 323-337. 
[30] J. N. Kapur, Generalized entropy of order a and type $\beta$. Math. Seminar, 4 (1967), 78-94.

[31] M. Ahsanullah, G. G. Hamedani, Characterizations of certain continuous univariate distributions based on the conditional distribution of generalized order statistics. Pak. J. Stat. 28 (2012), 253-258.

[32] M. Ahsanullah, Characterizations of Univariate Continuous Distributions. Atlantis Press, Paris, France 2017.

[33] M. Ahsanullah, M. Shakil, M. B. Golam Kibria, Characterizations of Continuous Distributions by Truncated Moment. J. Mod. Appl. Stat. Meth. 1(15) (2016), 316-331.

[34] M. A. Awad, J. A. Alawneh, Application of Entropy to life-Time Model. IMA J. Math. Control Inform. 4(1987), 143-147.

[35] M. C. Jones, Families of distributions arising from distributions of order statistics. Test, 13(2004), 143.

[36] G.M. Giorgi, S. Nadarajah, Bonferroni and Gini indices for various parametric families of distributions, METRON. 68 (2010), 23-46.

[37] M. H. Barakat, H. Y. Abdelkader, Computing the moments of order statistics from non-identical random variables. Stat. Meth. Appl. 13 (2004), 15-26.

[38] M. I. Kamien, N.L. Schwartz, A generalized hazard rate, Econ. Lett. 5(3) (1980), 245-249.

[39] M. Mitra, S. K. Basu, On some properties of the bathtub failure rate family of life distributions. Microelectron. Reliab. 36(5) (1996), 679-684.

[40] M. R. Fazlollah, An Introduction to Information Theory. McGraw-Hill, New York, 1961.

[41] M. M. Ristić, B. V. Popović, S. Nadarajah, Libby and Novick's generalized beta exponential distribution, J. Stat. Comput. Simul. 85 (2015), 740-761.

[42] N. Eugene, C. Lee, Felix Famoye, Beta-normal distribution and its application. Commun. Stat. Theory Meth. 31 (2002), 497-512.

[43] N. Pushkarna, J. Saran, and R. Tiwari, Bonferroni and Gini Indices and Recurrence Relation for Moments Progressive Type-II Right Censored Order Statistics from Marshall-Olkin Exponential Distribution. J. Stat. Theory Appl. 12(3) (2013), 306-320.

[44] P. T. Gia, N. Turkkan, Determine of the Beta Distribution form its Lorenz Curve. Math. Comput. Model. 16(2) (1992), 73-84.

[45] R Core Team, R A language and environment for statistical computing. Austria, Vienna: R Foundation for Statistical Computing, 2013.

[46] R. D. Gupta, D. Kundu, Generalized exponential distributions, Aust NZ J Stat. 41 (1999), 173-188.

[47] R. S. Varma, Generalizations of Renyi's entropy of order a. J. Math. Sci. 1 (1966), 34-48. 
[48] S. A. Hasnain, Z. Iqbal, M. Ahmad, One Exponentiated Moment Exponential Distribution. Pak. J. Stat. 31(2) (2015), 267-280.

[49] S. Arimoto, Information theoretical considerations on estimation theory. Inform. Control, 73 (1971), 181-190.

[50] S. Kullback, Information Theory and Statistics, Wiley, NY, 1959.

[51] S.M. Sunoj, P.G. Sankaran, S.S. Maya, Characterizations of Life Distributions Using Conditional Expectations of Doubly (Interval) Truncated Random Variables, Commun. Stat. - Theory Meth. 38 (2009) 1441-1452.

[52] S. Nadarajah, A. K. Gupta, The beta Fréchet distribution. Far East Theor. Stat. 14 (2004),15-24.

[53] S. Nadarajah, S. Kotz, The beta exponential distribution. Reliab. Eng. Syst. Safe. 91 (2006), 689-697.

[54] S. Nadarajah, S. Kotz, The beta Gumbel distribution. Math Probl. Eng. 10 (2004), 323-332.

[55] S. Pundir, S. Arora, K. Jain, Bonferroni Curve and the related Statistical Inference. Stat. Probab. Lett. 75(2005), 140-150.

[56] T. M. Cover, J. A. Thomas, Elements of Information Theory, John Wiley, New York, 1991.

[57] U. N. Nair, G. P. Sankaran, Properties of a Mean Residual Life Function Arising from Renewal Theory. Naval Res. Logist. 57 (2010), 373-379.

[58] V. Mameli, M. Musio, A new generalization of the skew-normal distribution: the beta skew-normal. Commun. Stat. - Theory Meth. 42 (2013) 2229-2244.

[59] V. Mameli, The Kumaraswamy skew-normal distribution, Stat. Probab. Lett. 104 (2015), 75-81.

[60] V. Mameli, Two generalizations of the skew-normal distribution and two variants of McCarthys Theo- rem. Doctoral dissertation, Cagliari University, Italy, 2012.

[61] V. Dardanoni, A. Forcina, Inference for Lorenz Curve orderings. Econ. J. 2 (1999), 49-75.

[62] W. F. Sharpe, Investments, Prentice Hall, Englewood Cliffs, 1985. 